\title{
Prevalência e fatores associados a defeitos de desenvolvimento do esmalte em crianças de 5 anos de idade matriculadas em creches na cidade de Teresina, Brasil
}

\section{Prevalence and factors associated with developmental defects of enamel in five-year-old children enrolled in preschools in the city of Teresina, Brazil}

\author{
Natália Silva Andrade (D), Samille Rodrigues Aquino² (D), \\ Isaac Torres dos Santos ${ }^{3}$ (D), Otacílio Batista de Sousa Nétto ${ }^{4}$ (D), Marcoeli Silva Moura ${ }^{3}$ (D), \\ Lúcia de Fátima Almeida de Deus Moura ${ }^{3}$ (D) Marina de Deus Moura de Lima ${ }^{3}$ (D) \\ 'Departamento de Odontologia, Universidade Federal de Sergipe - Lagarto (SE), Brasil. \\ ${ }^{2}$ Universidade Federal do Piauí - Teresina (PI), Brasil. \\ ${ }^{3}$ Programa de Pós-graduação em Odontologia, Universidade Federal do Piauí - Teresina (PI), Brasil. \\ ${ }^{4}$ Curso de Graduação em Odontologia, Universidade Federal do Piauí - Teresina (PI), Brasil.
}

Como citar: Andrade NS, Aquino SR, Santos IT, Souza Nétto OB, Moura MS, Moura LFAD, et al. Título completo do artigo no primeiro idioma. Cad Saúde Colet, 2021;29(4):528-537. https://doi.org/

\section{Resumo}

Introdução: Crianças com defeitos de desenvolvimento de esmalte (DDE) são mais suscetíveis à hipersensibilidade dentinária, maloclusões e dificuldades relativas à adesão de materiais restauradores. $\mathrm{O}$ conhecimento sobre a prevalência de DDE e seus fatores associados permite aos profissionais obter maior compreensão desse problema de saúde bucal, minimizando, dessa forma, as sequelas e melhorando a saúde bucal e a qualidade de vida dos indivíduos afetados. Objetivo: Determinar a prevalência de DDE e seus fatores associados na dentição decídua. Método: Trata-se de estudo transversal com amostra probabilística de 566 pré-escolares de 5 anos de idade, matriculados em creches públicas e privadas de Teresina, Piauí, Brasil. Foi aplicado questionário socioeconômico e de história médica da criança aos responsáveis. Os exames clínicos foram conduzidos por um único examinador (kappa = 0,93 para DDE e 0,86 para cárie) que utilizou os índices DDE modificado e ceo-d. Foi realizada a análise descritiva dos dados, além dos testes qui-quadrado e exato de Fisher e a regressão de Poisson, considerando como significativo $p \leq 0,05$. Resultados: A prevalência de DDE foi de 33,7\%. A média de dentes com DDE por criança foi de $3,12 \pm 2,23$. O tipo mais prevalente de DDE foi opacidade demarcada (9,5\%). Os segundos molares superiores foram os dentes mais afetados (11,9\%). Houve associação entre DDE e estudar em creche pública $(p=0,026)$ e cárie $(p=0,012)$. Crianças com experiência de cárie apresentaram maior prevalência de DDE (RP: 1,29; IC95\%: 1,01-1,64). Conclusão: A prevalência de DDE na dentição decídua foi de $33,7 \%$ e se mostrou associada à experiência de cárie.

Palavras-Chave: esmalte dentário; cárie; prevalência; fatores de risco; dentição decídua.

\begin{abstract}
Background: Children with Developmental Defects of Enamel (DDE) are more susceptible to dentin hypersensitivity, malocclusions, and difficulties related to the adhesion of restorative materials. Knowledge about the prevalence and factors associated with DDE allows professionals to gain a greater
\end{abstract}

Trabalho realizado em Teresina (PI), Brasil.

Correspondência: Natália Silva Andrade. E-mail: nataliaa.odonto@gmail.com

Fonte de Financiamento: Fundação de Amparo à Pesquisa do Estado do Piaú́ (FAPEPI), edital PPSUS n003/2015.

Conflito de Interesses: nada a declarar.

Recebido em: Jan. 20, 2019. Aprovado em: Jul. 20, 2020
Este é um artigo publicado em acesso aberto (Open Access) sob a licença Creative Commons Attribution, que permite uso, distribuição e reprodução em qualquer meio, sem restrições desde que o trabalho original seja corretamente citado. 
understanding of this oral health problem, minimizing sequelae, improving oral health and quality of life of affected individuals. Objective: To determine the prevalence and factors associated with DDE in primary dentition. Method: This is a cross-sectional study assessing a random sample of 566 preschool five-year-old children, enrolled in public and private preschools in Teresina, Piauí, Brazil. It was applied the socioeconomic and medical history of the child questionnaire for the parents or guardians. Clinical examinations were conducted by a single examiner (kappa $=0.93$ for DDE and 0.86 for caries) that used the modified DDE index and dmft. Descriptive analysis, chi-square and Fisher's exact tests, and Poisson regression were performed, considering significant $p \leq 0.05$. Results: the prevalence of DDE was $33.7 \%$. The mean DDE teeth per child was $3.12 \pm 2.23$. The most prevalent type of DDE was demarcated opacity (9.5\%). The second molars were the most affected teeth (11.9\%). There was an association between DDE and study at public preschool $(p=0.026)$ and dental caries $(p=0.012)$. Children with experience of caries were more likely to DDE (PR: $1.29 ; 95 \% \mathrm{Cl}: 1.01$ to 1.64). Conclusion: prevalence of DDE in the primary dentition was $33.7 \%$ and was associated with caries experience.

Keywords: dental enamel; dental caries; prevalence; risk factors; primary dentition.

\section{INTRODUCCÃO}

Defeitos de desenvolvimento de esmalte (DDE) são alterações resultantes de distúrbios no processo da amelogênese ${ }^{1,2}$ e podem ser classificados como qualitativos (opacidades difusa ou demarcada) e/ou quantitativos (hipoplasia) ${ }^{1}$. Para Federação Dentária Internacional (FDI), a opacidade demarcada é uma alteração na translucidez do esmalte, com bordos definidos a partir do esmalte adjacente normal, com coloração branca, bege, amarela ou marrom. A opacidade difusa é uma anormalidade na translucidez do esmalte, na qual não existe um limite nítido entre o esmalte normal adjacente e a opacidade referida. A hipoplasia é uma redução localizada da espessura de esmalte com ou sem exposição de dentina'.

A prevalência de DDE na dentição decídua relatada em estudos internacionais, os quais utilizaram os critérios de diagnóstico da FDI, varia entre 5,3 e 74,2\% $\%^{3,4}$. Em estudos brasileiros, essa prevalência varia de $22,8 \%$ na região Sudeste a $78,9 \%$ na região Nordeste ${ }^{5,6}$. Essa grande variação pode estar relacionada, entre outros aspectos, às características da amostra estudada, como região, faixa etária, critérios diagnósticos e metodologias aplicadas nas pesquisas ${ }^{7,8}$. A etiologia de DDE na dentição decídua permanece controversa ${ }^{9}$. Acredita-se que seja multifatorial, influenciada por fatores locais, ambientais sistêmicos e/ou hereditários. Como os ameloblastos são células extremamente sensíveis às variações do ambiente, pode ter como consequência o surgimento de defeitos no esmalte ${ }^{2,9,10}$. Condições sociodemográficas desfavoráveis ${ }^{6}$, alterações nutricionais ${ }^{11}$, problemas gestacionais, prematuridade, baixo peso ao nascer e doenças da primeira infância têm sido associados a um risco aumentado de DDE na dentição decídua ${ }^{10,12}$.

Revisões sistemáticas têm demonstrado que crianças com DDE são mais suscetíveis à cárie. Ambas as condições têm alta prevalência na primeira infância, e a cárie é considerada um problema de saúde pública ${ }^{13,14}$. Além disso, indivíduos com DDE são mais suscetíveis à hipersensibilidade dentinária, maloclusões e dificuldades relativas à adesão de materiais, levando a necessidade de substituições cada vez mais frequentes de restaurações ${ }^{6,9,15}$. A susceptibilidade à cárie pode ser consequência das alterações morfológicas e estruturais, como alterações de cor, menor conteúdo mineral, irregularidades na superfície dentária e disposição frouxa dos prismas de esmalte, levando ao maior acúmulo de biofilme, dificuldade de higienização, perda de estrutura dentária e alterações estéticas ${ }^{9,13}$.

O conhecimento sobre a prevalência e a distribuição de DDE e seus fatores associados permite aos profissionais obter maior compreensão desse problema de saúde bucal. O diagnóstico, idealmente precoce, possibilitaria o estabelecimento de medidas de prevenção secundária e intervenção adequadas, a fim de minimizar as sequelas e melhorar a saúde bucal e a qualidade de vida dos indivíduos afetados ${ }^{16,17}$.

Revisões sistemáticas têm demonstrado uma associação entre a presença de DDE e a cárie, o problema de saúde bucal mais prevalente na primeira infância ${ }^{15,18}$. Sendo o Brasil um país de dimensões continentais, com diferenças regionais significativas, e sendo o Piauí um dos estados com menor índice de desenvolvimento humano, ainda não foi estudada e discutida a prevalência de DDE e de seus fatores associados em crianças de idade pré-escolar de Teresina, 
Piauí, Brasil. Por isso, o objetivo deste trabalho foi realizar um estudo epidemiológico para determinar a prevalência de DDE e de seus fatores associados na dentição decídua em crianças com 5 anos de idade.

\section{MÉTODO}

\section{Considerações éticas}

Este estudo observacional transversal foi aprovado pelo Comitê de Ética em Pesquisa da Universidade Federal do Piauí (UFPI), Brasil. Os responsáveis pelas crianças concordaram em participar da pesquisa e assinaram o Termo de Consentimento Livre e Esclarecido (TCLE), e as crianças assinaram o Termo de Assentimento Livre e Esclarecido (TALE), obedecendo às diretrizes que regulamentam as pesquisas envolvendo seres humanos, previstas na Resolução n 466/12, do Conselho Nacional de Saúde.

\section{População e amostra}

A população do estudo foi constituída por pré-escolares com idade de 5 anos, do município de Teresina, Piauí, Brasil. A amostra foi do tipo estratificada, simples e aleatória, calculada utilizando a equação $n=z^{2}$.p.(1-p)/e $e^{2}$, em que:" $z$ "é o quantil da distribuição normal (para o intervalo de confiança de $95 \%$, tem-se $z=1,96$ ); " $p$ " é a variação estimada para DDE $(30 \%)^{17}$; e "e" é a margem de erro considerada (5\%). Em seguida, foi aplicada a correção de Cochran para populações finitas, $\mathrm{n}=\mathrm{n} 0 /(1+\mathrm{n} 0 / \mathrm{N})$, em que: $\mathrm{n} 0$ é o tamanho inicial da amostra; e N é o tamanho da população (7.792 pré-escolares). Foi obtida amostra mínima para o desenvolvimento do estudo de 345 crianças. Por se tratar de uma amostra em múltiplos estágios, foi corrigido o efeito do desenho por um fator de 1,5 (345 x 1,5=518). Para minimizar possíveis perdas, foi aumentado o tamanho da amostra em $10 \%$, sendo obtida uma amostra de $570(518+52=570)$ pré-escolares.

Foi realizado sorteio aleatório de 40 instituições de ensino infantil (total $=302$ ) proporcionalmente à superintendência regional em que estavam localizadas (norte, sul, leste e sudeste) e ao tipo de pré-escola (pública ou privada), a partir de listas fornecidas pela Secretaria Municipal de Educação e Sindicato de Escolas Particulares do município. Para melhor aleatorização da amostra, em cada instituição as crianças foram sorteadas a partir das listas de frequência escolar.

\section{Critérios de inclusão e exclusão}

Foram incluídos na amostra pré-escolares com 5 anos de idade, que possuíam dentição decídua completa na data do exame clínico intrabucal. Foram considerados inelegíveis para o estudo crianças com necessidades especiais, doenças sistêmicas ou não cooperativas durante o exame clínico.

\section{Calibração}

O exercício de calibração foi realizado em duas fases. A fase teórico-prática foi coordenada por especialista em odontopediatria (MDML) com experiência em pesquisas sobre o tema e envolveu a discussão dos critérios de diagnóstico de DDE e cárie ${ }^{1,19}$. Foram feitas discussão e análise de 20 fotografias de dentes com e sem DDE e/ou cárie. Após examinadora (NSA) e especialista concordarem em $80 \%$ das avaliações, foi iniciada a segunda fase da calibração. A fase clínica foi conduzida durante o estudo-piloto, no qual foi determinada a concordância intraexaminador (índice Kappa =0,86 para cárie e 0,93 para DDE). Para tanto, foi realizada avaliação clínica de 60 crianças em dois momentos, com intervalos mínimos 15 dias entre os dois exames clínicos. 


\section{Estudo-piloto}

Foi realizado estudo-piloto com 60 crianças em três creches (duas públicas e uma privada), que não foram incluídas na amostra final, com o objetivo de adequar os métodos da pesquisa (questionário e avaliação odontológica), calibração e preparação do examinador. Não houve necessidade de alterações nos métodos propostos.

\section{Coleta de dados}

Os dados foram coletados no período de outubro a dezembro de 2014, por meio de questionário sociodemográfico e de fatores associados, aplicados aos responsáveis pelas crianças, seguidos de exame clínico intrabucal. No primeiro momento, foi solicitada a autorização da instituição de ensino para a realização da pesquisa e o posterior sorteio das crianças. Em relação às crianças sorteadas, foram enviados questionários para seus responsáveis com o TCLE, e, na segunda visita à instituição, elas assinaram o TALE antes da realização do exame clínico.

\section{Questionário sociodemográfico e de fatores potencialmente associados a DDE}

$O$ instrumento utilizado e desenvolvido exclusivamente para este estudo continha perguntas sobre sexo, renda familiar (categorizada com base no salário mínimo mensal brasileiro, de $\mathrm{R} \$ 724,00$, relativo ao ano de 2014), escolaridade da mãe e do pai (em anos de estudo formal, com ponto de corte de 8 anos, correspondente ao nível de educação primária no Brasil), tipo de pré-escola (pública ou privada), rede de abastecimento de água na residência e condições clínicas possivelmente relacionadas à etiologia de DDE selecionadas a partir de outros estudos já publicados na literatura (febre ou infecção durante a gestação, prematuridade e doenças da primeira infância).

\section{Exame clínico dentário}

O exame clínico foi realizado para avaliar a experiência de cárie, a presença e o tipo de DDE. No diagnóstico de cárie, foi utilizado o índice ceo-d, que expressa o somatório do número de dentes cariados, perdidos por causa de cárie e obturados ${ }^{18}$. 0 índice DDE modificado, preconizado pela FDI (1992), foi utilizado para avaliação da presença de opacidade demarcada, opacidade difusa, hipoplasia, combinações ou outros tipos de defeitos ${ }^{1}$.

Essa avaliação clínica foi conduzida em sala de aula na própria instituição de ensino por um único examinador previamente calibrado. Foram concedidas orientações sobre cuidados com a saúde bucal e doados kits de higiene bucal. Antes do exame, foi realizada higienização dos dentes com escova dental e dentifrício fluoretado. Foi utilizada gaze estéril para secar os dentes, e o exame dentário foi feito com o auxílio de espelho bucal plano (Golgran ${ }^{\oplus}$, São Paulo, Brasil) e sonda CPI (Trinity ${ }^{\circledR}$, Pará, Brasil). As crianças foram examinadas sob iluminação artificial (luminária de mesa modelo Pelicano ${ }^{\oplus}$ - Startec com 127V, São Paulo, Brasil), em posição simplificada com a cabeça sobre as pernas do examinador. Crianças com diagnóstico de doenças bucais foram encaminhadas para o atendimento odontológico na Clínica Odontológica Infantil da UFPI.

\section{Análise estatística}

Os dados foram analisados no programa Statistical Package for the Social Sciences (SPSS ${ }^{\circledR}$ for Windows, versão 20.0, SPSS Inc., Chicago, IL, Estados Unidos). Foi realizada a análise descritiva dos dados. O poder da amostra foi calculado para prevalência estimada de DDE. As associações entre defeitos de esmalte e variáveis independentes foram determinadas pelos testes qui-quadrado $\left(X^{2}\right)$ e exato de Fisher. $\mathrm{Na}$ análise multivariada, foram incluídas as variáveis que apresentaram valor de $p \leq 0,20$ na análise bivariada (renda familiar, tipo de pré-escola, febre alta, desidratação/diarreia e cárie). A regressão de Poisson com variância 
robusta (matriz de covariância com estimador robusto) foi utilizada para análise dos fatores associados a DDE, com a ausência de defeitos de esmalte sendo utilizada como categoria de referência. Os resultados foram expressos em razão de prevalência (RP) não ajustada e ajustada, com intervalo de confiança de 95\% (IC95\%). Permaneceram no modelo as associações que alcançaram valor de $p<0,05$ (teste de Wald). Em todas as análises, foi considerado o nível de significância $a=5 \%$.

\section{RESULTADOS}

A amostra final foi constituída por 566 crianças (99,3\%). No dia do exame, 4 crianças $(0,7 \%)$ que tinham diagnóstico de transtorno do espectro autista e não cooperaram para a realização do exame clínico bucal não foram incluídas na pesquisa. A prevalência de defeitos do esmalte foi de $33,7 \%$ e a experiência de cárie foi de $50,2 \%$. A média de dentes com DDE por criança foi de $3,12 \pm 2,23$, enquanto o índice ceo-d médio foi de $2,06 \pm 3,09$, dos quais $1,78 \pm 2,83$ era cariado, 0,08 $\pm 0,40$ extraído e 0,17 $\pm 0,67$ obturado.

Associações entre DDE e variáveis relacionadas aos dados sociodemográficos e à etiologia dos defeitos de esmalte estão descritas na Tabela 1. Experiência de cárie e estudar em pré-escola pública foram significativamente associados com DDE ( $p=0,026$ e $p=0,012$, respectivamente). No modelo de regressão ajustado, somente experiência de cárie (RP: 1,29; IC95\%: 1,01-1,64) se manteve associada com DDE na dentição decídua, independentemente de outras variáveis (Tabela 2 ).

Quanto à distribuição dos tipos de DDE e combinações, opacidade demarcada foi o tipo de DDE mais prevalente (9,5\%), seguido de hipoplasia (8,3\%), opacidade difusa $(8,1 \%)$, combinação de opacidade difusa com demarcada $(3,2 \%)$ e de opacidade difusa com hipoplasia (2,5\%). Os DDE foram mais frequentes no arco superior do que no arco inferior (Gráficos 1 e 2). Os dentes mais afetados foram os segundos molares superiores (12\%), seguidos pelos segundos molares e caninos inferiores direitos $(8,1 \%)$.

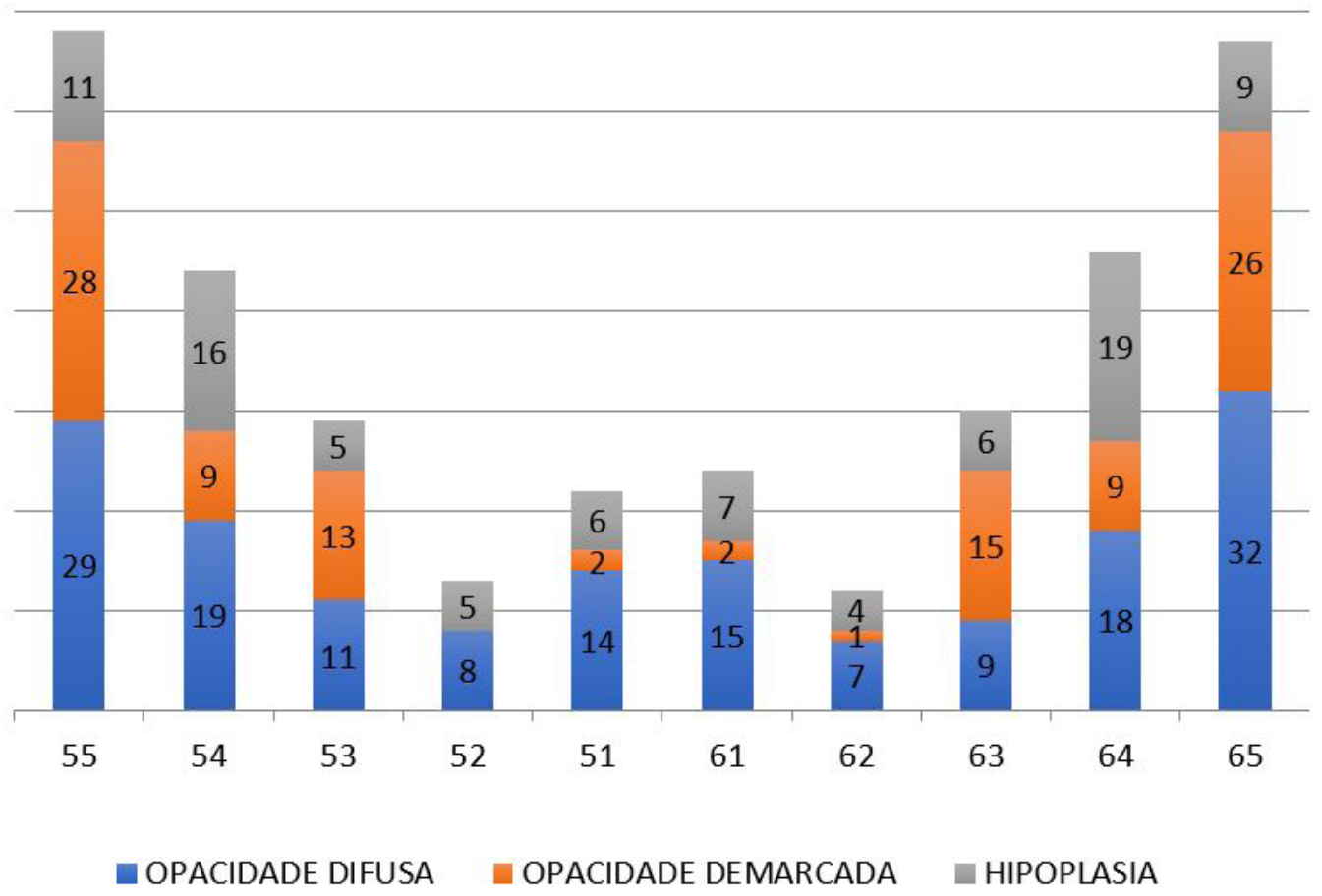

Gráfico 1. Distribuição dos tipos de defeitos de desenvolvimento de esmalte em dentes decíduos na maxila (números absolutos). Teresina, Piauí-PI, Brasil, 2014. 
Tabela 1. Análise bivariada entre defeitos de desenvolvimento de esmalte (DDE), fatores sociodemográficos e história médica de crianças com 5 anos de idade matriculadas em creches de Teresina, Piauí-PI, Brasil, 2014.

\begin{tabular}{|c|c|c|c|c|}
\hline & Presença de DDE & Ausência de DDE & \multirow{2}{*}{$\mathbf{P}^{*}$} & \multirow{2}{*}{$\begin{array}{c}\text { RP bruta } \\
\text { RP (IC95\%) } \\
\end{array}$} \\
\hline & $\mathrm{n}(\%)$ & n (\%) & & \\
\hline Sexo & & & 0,430 & \\
\hline Masculino & $106(35,2)$ & $195(64,8)$ & & $1,15(0,81-1,63)$ \\
\hline Feminino & $85(32,1)$ & $180(67,9)$ & & \\
\hline Renda familia & & & 0,084 & \\
\hline$\leq 2 \mathrm{SM}$ & $138(36,1)$ & $244(63,9)$ & & $1,39(0,95-2,04)$ \\
\hline$>2 S M$ & $53(28,8)$ & $131(71,2)$ & & \\
\hline \multicolumn{2}{|c|}{ Escolaridade da mãe (anos de estudo) } & & 0,939 & \\
\hline$\leq 8$ & $51(34,0)$ & $99(66,0)$ & & $1,01(0,68-1,50)$ \\
\hline$>8$ & $140(33,7)$ & $276(66,3)$ & & \\
\hline \multicolumn{2}{|c|}{ Escolaridade do pai (anos de estudo) } & & 0,926 & \\
\hline$\leq 8$ & $68(33,5)$ & $135(66,5)$ & & $0,98(0,68-1,41)$ \\
\hline$>8$ & $123(33,9)$ & $240(66,1)$ & & \\
\hline Tipo de pré-e & & & 0,026 & \\
\hline Pública & $140(36,8)$ & $240(63,2)$ & & $1,54(1,05-2,26)$ \\
\hline Privada & $51(27,4)$ & $135(72,6)$ & & \\
\hline \multicolumn{2}{|c|}{ História de febre e/ou infecção gestacional } & & $0,339^{*}$ & \\
\hline Sim & $25(39,1)$ & $39(60,9)$ & & $1,29(0,76-2,21)$ \\
\hline Não & $166(33,1)$ & $336(66,9)$ & & \\
\hline \multicolumn{2}{|c|}{ Prematuridade } & & $0,327^{*}$ & \\
\hline Sim & $20(40,0)$ & $30(60,0)$ & & $1,34(0,74-2,43)$ \\
\hline Não & $171(33,1)$ & $345(66,9)$ & & \\
\hline \multicolumn{2}{|l|}{ Sarampo } & & $0,439^{* *}$ & \\
\hline Sim & $0(0,0)$ & $2(100,0)$ & & $1,51(1,42-1,60)$ \\
\hline Não & $191(33,9)$ & $373(66,1)$ & & \\
\hline \multicolumn{2}{|l|}{ Varicela } & & $0,893^{*}$ & \\
\hline Sim & $35(34,3)$ & $67(65,7)$ & & $1,03(0,65-1,62)$ \\
\hline Não & $156(33,6)$ & $308(66,4)$ & & \\
\hline \multicolumn{2}{|l|}{ Pneumonia } & & $0,505^{*}$ & \\
\hline Sim & $19(38,0)$ & $31(62,0)$ & & $1,22(0,67-2,23)$ \\
\hline Não & $172(33,3)$ & $344(66,7)$ & & \\
\hline \multicolumn{2}{|l|}{ Caxumba } & & $0,585^{* *}$ & \\
\hline Sim & $1(25,0)$ & $3(75,0)$ & & $0,65(0,07-6,31)$ \\
\hline Não & $190(33,8)$ & $372(66,2)$ & & \\
\hline \multicolumn{2}{|l|}{ Febre alta } & & $0,138^{*}$ & \\
\hline Sim & $34(28,1)$ & $87(71,9)$ & & $0,71(0,46-1,11)$ \\
\hline Não & $157(35,3)$ & $288(64,7)$ & & \\
\hline \multicolumn{2}{|c|}{ Desidratação/diarreia } & & $0,155^{*}$ & \\
\hline $\operatorname{Sim}$ & $30(41,1)$ & $43(58,9)$ & & $1,43(0,87-2,37)$ \\
\hline Não & $161(32,7)$ & $332(67,3)$ & & \\
\hline \multicolumn{2}{|l|}{ Outra doença } & & $0,867^{*}$ & \\
\hline Sim & $21(32,8)$ & $43(67,2)$ & & $0,95(0,54-1,66)$ \\
\hline Não & $170(33,9)$ & $332(66,1)$ & & \\
\hline Total & $191(33,7)$ & $375(66,3)$ & & \\
\hline
\end{tabular}

SM = salários mínimos; RP bruta = razão de prevalência bruta; $I C 95 \%$ = intervalo de confiança de $95 \%$; *teste qui-quadrado $\left(X^{2}\right)$; **teste exato de Fisher 
Tabela 2. Presença de defeitos de desenvolvimento do esmalte (DDE) e experiência de cárie de crianças com 5 anos de idade matriculadas em creches de Teresina, Piauí-PI, Brasil, e modelo final de regressão de Poisson para o grupo com DDE. 2014.

\begin{tabular}{|c|c|c|c|}
\hline \multirow{2}{*}{ Experiência de cárie } & Presença de DDE & Ausência de DDE & \multirow{2}{*}{$\mathbf{p}^{*}$} \\
\hline & n (\%) & n (\%) & \\
\hline Não & $81(42,4)$ & $201(53,6)$ & 0,012 \\
\hline Sim & $110(57,6)$ & $174(46,4)$ & \\
\hline Variável & RP bruta (IC 95\%) & RP ajustada (IC 95\%) & $\mathbf{P}$ \\
\hline \multicolumn{4}{|l|}{ Experiência de cárie } \\
\hline Não & 1 & 1 & 0,040 \\
\hline Sim & $1,35(1,07-1,71)$ & $1,29(1,01-1,64)$ & \\
\hline
\end{tabular}

RP bruta = razão de prevalência bruta; RP ajustada = razão de prevalência ajustada; IC95\% = intervalo de confiança de $95 \%$; $\mathrm{P}=$ probabilidade do teste de Wald qui-quadrado; *teste qui-quadrado $\left(\mathrm{X}^{2}\right)$

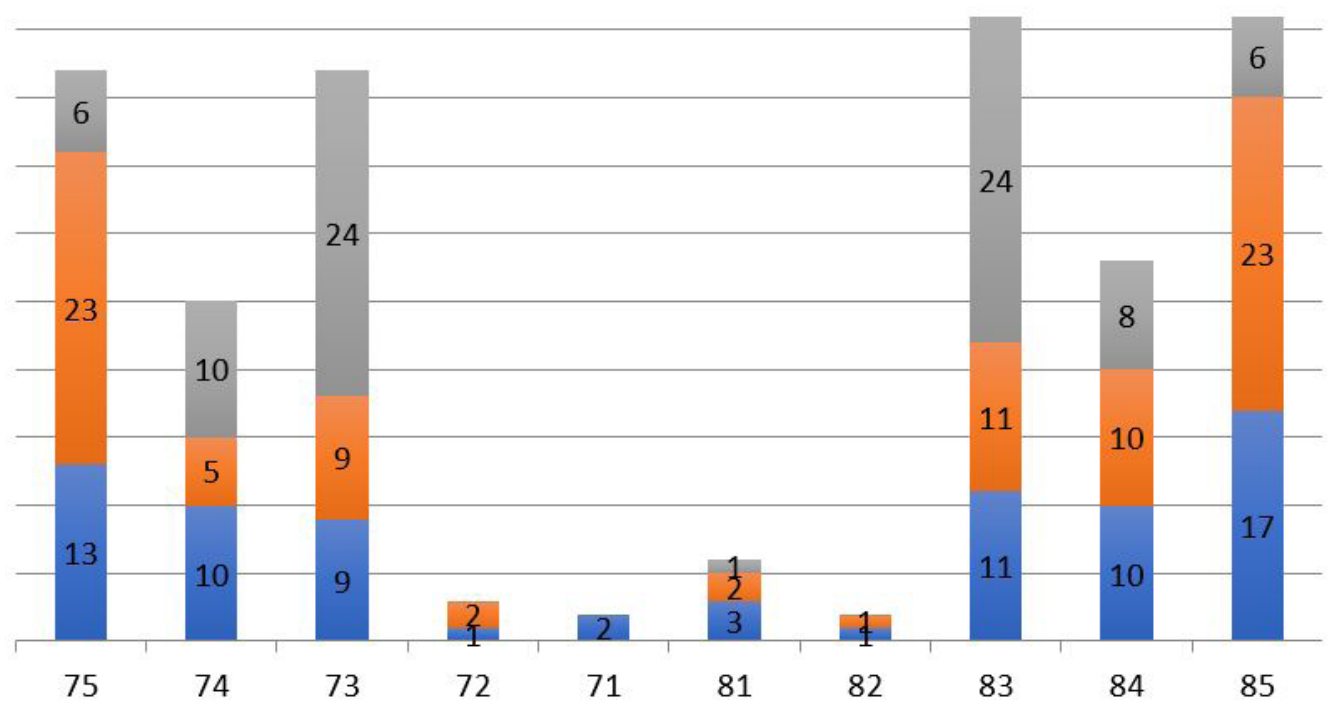

OPACIDADE DIFUSA OOPACIDADE DEMARCADA HIPOPLASIA

Gráfico 2. Distribuição dos tipos de defeitos de desenvolvimento de esmalte em dentes decíduos na mandíbula (números absolutos). Teresina, Piauí-PI, Brasil, 2014.

\section{DISCUSSÃO}

A prevalência de DDE em dentes decíduos foi expressiva e semelhante a outros estudos brasileiros ${ }^{15,17}$. Opacidade demarcada foi o tipo mais frequente, corroborando resultados de estudos prévios ${ }^{17,19-21}$. Resultados divergentes foram apontados em pesquisas de outras regiões brasileiras e de outros países, que relataram maior ocorrência de opacidade difusa ${ }^{6-8,11}$. Essa diferença pode ser justificada pela dificuldade no diagnóstico de delimitação visível entre o esmalte afetado e o normal adjacente ${ }^{1}$, provocada pela coloração mais esbranquiçada dos dentes decíduos. Além disso, existem diferenças metodológicas utilizadas para a avaliação do esmalte dentário ${ }^{6,7,11}$, assim como diferenças regionais, especialmente no Brasil, por ser um país com grandes dimensões territoriais e diversidade socioeconômica e cultural. Os defeitos foram mais frequentes em dentes superiores, talvez por estarem relacionados à calcificação mais lenta ou início mais precoce da mineralização nesses dentes ${ }^{22,23}$. 
A associação entre DDE e experiência de cárie em dentes decíduos foi observada neste e em outros estudos ${ }^{6,15}$. A estrutura alterada do esmalte dentário facilita a adesão e a colonização de bactérias cariogênicas ${ }^{6,15,24}$, predispondo ao acúmulo de biofilme dental e consequente desenvolvimento de cárie e doença periodontal. Sendo a cárie uma condição passível de prevenção, é essencial identificar precocemente crianças com DDE em virtude de essa condição ser fator de risco para o desencadeamento da doença ${ }^{13,15}$.

Apesar da associação entre DDE e cárie ${ }^{14}$, comparações entre os estudos devem ser realizadas com cautela. Investigações transversais anteriores apresentam diferenças metodológicas em relação ao presente estudo, tais como ferramentas utilizadas para o diagnóstico de DDE, tipos de defeitos estudados, além de fatores específicos das populações investigadas, por exemplo, genética, etnia, condição socioeconômica e demográfica ${ }^{25-27}$. Essa associação na dentição decídua pode ser modulada por interação entre hábitos familiares de ingestão de açúcares e hábitos de higiene bucal com o uso de dentifrícios fluoretados, além de predisposições individuais ${ }^{16,28}$.

O tratamento de cárie exige um alto custo econômico tanto em níveis individuais como em níveis do sistema de saúde ${ }^{29}$. A cárie não tratada em dentes decíduos é a principal causa de dor de origem odontológica entre crianças e pode ser agravada na presença de $\mathrm{DDE}$, afetando o medo odontológico e levando ao receio em procurar o tratamento odontológico ${ }^{30}$. Esses fatores podem impactar negativamente a qualidade de vida relacionada à saúde bucal de crianças e seus responsáveis ${ }^{13}$.

Crianças matriculadas em creches públicas apresentaram maior prevalência de DDE, contudo a associação não permaneceu no modelo final de regressão. A relação entre fatores sociais e problemas bucais, incluindo DDE, tem sido sugerida" ${ }^{11}$. Nos países em desenvolvimento, como o Brasil, crianças de famílias de baixa renda são, geralmente, sujeitas a problemas nutricionais e maior incidência de infecções. Assim, desigualdades sociais e iniquidades em saúde, mais do que qualquer problema de saúde coletiva, são consideradas as causas distais e proximais dos problemas de saúde a nível populacional e podem ter impacto importante sobre a saúde bucal ${ }^{17}$. A cidade de Teresina é a capital do Piauí e possui melhores condições de vida (saneamento básico, educação etc.), contudo o estado é um dos mais pobres do país.

Não foram observadas associações entre os fatores pré-, peri- e pós-natais estudados e DDE. Apesar da etiologia não esclarecida, DDE são causados por perturbações durante desenvolvimento do esmalte dentário, e elevada prevalência na dentição decídua demonstra vulnerabilidade a alterações no ambiente pré-, peri- e pós-natal ${ }^{9}$. A ausência de associação neste estudo pode ser explicada pelo pequeno número de crianças que apresentaram as condições investigadas. Além disso, as informações obtidas do questionário aplicado aos responsáveis estão sujeitas a viés de memória.

A produção de conhecimento sobre a prevalência e a distribuição de DDE e fatores associados e sua divulgação acadêmica, científica e para as comunidades de profissionais e de usuários dos diferentes serviços de saúde (públicos e privados) potencializam o estabelecimento de diagnósticos precoces, medidas de prevenção e intervenção adequadas para esses defeitos ${ }^{16}$. As intervenções sobre os DDE podem ser iniciadas no pré-natal ampliado por meio do acompanhamento sistemático do ciclo gravídico-puerperal por equipe multidisciplinar, interprofissional, com interconsultas frequentes, para minimizar problemas de saúde tanto na mãe como na criança ${ }^{10}$. Recomenda-se que a criança seja examinada por um cirurgião-dentista antes dos 12 meses de idade, visando, principalmente, ao diagnóstico precoce ${ }^{16}$.

O manejo de pacientes com DDE na dentição decídua também inclui medidas de prevenção secundária para minimizar as sequelas ocasionadas por DDE, como uso de agentes remineralizantes, selantes e, em casos severos, restaurações com coroas de aço inoxidável para dentes posteriores e resina composta para dentes anteriores, avaliando o binômio custobenefício e as dificuldades intrínsecas do atendimento odontopediátrico ${ }^{9}$. Assim, são garantidas a existência e a disponibilidade de serviços odontológicos para grande parcela da população infantil, especialmente aquelas que frequentam os equipamentos sociais públicos.

O presente estudo apresenta algumas limitações. O delineamento transversal não permite a avaliação de causalidade. O índice DDE modificado preconizado pela FDI' avalia defeitos de 
esmalte de forma mais abrangente, não especificando tipos particulares de defeitos, como a hipomineralização de molares decíduos (HMD). Além disso, apenas lesões cariosas cavitadas foram avaliadas por meio do índice ceo-d, e o fato de não avaliar lesões de mancha branca pode ter levado à subestimação da prevalência de cárie.

Os resultados do presente estudo possibilitaram a melhor compreensão sobre a magnitude e a distribuição dos DDE na população estudada e sua associação com a cárie, representando um problema de saúde bucal frequente e que pode suscitar a necessidade de intervenções por parte do setor público. As intervenções em ambas as condições (DDE e cárie) visam ao diagnóstico precoce, ao estabelecimento de medidas preventivas primárias e secundárias, que vão desde o pré-natal odontológico até o acompanhamento do desenvolvimento da dentição decídua e a determinação de tratamentos adequados a cada caso, a fim de minimizar as sequelas e melhorar e/ou devolver a saúde bucal e a qualidade de vida dos indivíduos afetados.

A prevalência de DDE foi considerada alta, sendo associada à presença de cárie. Assim, são sugeridas abordagens que visem ao direcionamento de políticas públicas para grupos com DDE.

\section{AGRADECIMENTOS}

Este estudo teve apoio financeiro da Fundação de Amparo à Pesquisa do Estado do Piauí (FAPEPI), edital PPSUS nº03-2013/2015. Os autores agradecem às crianças, aos responsáveis, às escolas e à Secretaria Municipal de Educação de Teresina/PI, Brasil, que tornaram possível a realização deste estudo. Participaram como anotadores os discentes, Helly K. P. Soares, Ingred B. Lopes, Renara N. C. Silva, Lisanca Q. C. Carvalho, Brenda I. S. Mota e Hélvis Enri de S. Paz.

\section{REFERÊNCIAS}

1. Federation Dentaire Internationale. A review of the developmental defects of dental index (DDE index). Commission on Oral Health Research and Epidemiology. Report of an FDI Working Group. Int Dent J. 1992;42(6):411-26. PMid:1286924.

2. Jacobsen PE, Haubek D, Henriksen TB, Østergaard JR, Poulsen S. Developmental enamel defects in children born preterm: a systematic review. Eur J Oral Sci. 2014;122(1):7-14. http://dx.doi.org/10.1111/eos.12094. PMid:24164573.

3. Wagner Y. Developmental defects of enamel in primary teeth - findings of a regional German birth cohort study. BMC Oral Health. 2016;17(1):10. http://dx.doi.org/10.1186/s12903-016-0235-7. PMid:27430531.

4. Osorio-Tovar JP, Naranjo-Sierra MC, Rodríguez-Godoy M. Prevalence of developmental defects of the enamel in primary dentition in a bogotan population. Rev Salud Publica. 2016;18(6):963-75. PMid:30137179.

5. Corrêa-Faria P, Paixão-Gonçalves S, Paiva SM, Martins-Júnior PA, Vieira-Andrade RG, Marques LS, et al. Dental caries, but not malocclusion or developmental defects, negatively impacts preschoolers' quality of life. Int J Paediatr Dent. 2016;26(3):211-9. http://dx.doi.org/10.1111/ipd.12190. PMid:26173864.

6. Oliveira AF, Chaves AM, Rosenblatt A. The influence of enamel defects on the development of early childhood caries in a population with low socioeconomic status: a longitudinal study. Caries Res. 2006;40(4):296-302. http://dx.doi.org/10.1159/000093188. PMid:16741360.

7. Robles MJ, Ruiz M, Bravo-Perez M, González E, Peñalver MA. Prevalence of enamel defects in primary and permanent teeth in a group of schoolchildren from Granada (Spain). Med Oral Patol Oral Cir Bucal. 2013;18(2):e187-93. http://dx.doi.org/10.4317/medoral.18580. PMid:23229271.

8. Masumo R, Bårdsen A, Åstrøm AN. Developmental defects of enamel in primary teeth and association with early life course events: a study of 6-36 month old children in Manyara, Tanzania. BMC Oral Health. 2013;13(1):21. http://dx.doi.org/10.1186/1472-6831-13-21. PMid:23672512.

9. Salanitri S, Seow WK. Developmental enamel defects in the primary dentition: aetiology and clinical management. Aust Dent J. 2013;58(2):133-40. http://dx.doi.org/10.1111/adj.12039. PMid:23713631.

10. Corrêa-Faria P, Martins-Júnior PA, Vieira-Andrade RG, Marques LS, Ramos-Jorge ML. Perinatal factors with developmental defects of enamel in primary teeth: a case-control study. Braz Oral Res. 2013;27(4):363-8. http://dx.doi.org/10.1590/S1806-83242013005000017. PMid:23689469.

11. Massoni AC, Chaves AM, Rosenblatt A, Sampaio FC, Oliveira AF. Prevalence of enamel defects related to pre-, peri- and postnatal factors in a Brazilian population. Community Dent Health. 2009;26(3):143-9. PMid:19780354. 
12. Vargas-Ferreira F, Zeng J, Thomson WM, Peres MA, Demarco FF. Association between developmental defects of enamel and dental caries in schoolchildren. J Dent. 2014;42(5):540-6. http://dx.doi.org/10.1016/j. jdent.2014.02.010. PMid:24561341.

13. Costa FS, Silveira ER, Pinto GS, Nascimento GG, Thomson WM, Demarco FF. Developmental defects of enamel and dental caries in the primary dentition: a systematic review and meta-analysis. J Dent. 2017;60:1-7. http://dx.doi.org/10.1016/j.jdent.2017.03.006. PMid:28347809.

14. Vargas-Ferreira F, Salas MMS, Nascimento GG, Tarquinio SBC, Faggion CM Jr, Peres MA, et al. Association between developmental defects of enamel and dental caries: a systematic review and meta-analysis. J Dent. 2015;43(6):619-28. http://dx.doi.org/10.1016/j.jdent.2015.03.011. PMid:25862273.

15. Corrêa-Faria P, Paixão-Gonçalves S, Paiva SM, Pordeus IA, Marques LS, Ramos-Jorge ML. Association between developmental defects of enamel and early childhood caries: a cross-sectional study. Int J Paediatr Dent. 2015;25(2):103-9. http://dx.doi.org/10.1111/ipd.12105. PMid:24650107.

16. Seow WK. Developmental defects of enamel and dentine: challenges for basic science research and clinical management. Aust Dent J.2014;59(Suppl 1):143-54. http://dx.doi.org/10.1111/adj.12104. PMid:24164394.

17. Corrêa-Faria P, Martins-Júnior PA, Vieira-Andrade RG, Oliveira-Ferreira F, Marques LS, Ramos-Jorge ML. Developmental defects of enamel in primary teeth: prevalence and associated factors. Int J Paediatr Dent. 2013;23(3):173-9. http://dx.doi.org/10.1111/j.1365-263X.2012.01241.x. PMid:22548676.

18. World Health Organization. Oral health surveys, basics methods. 5th ed. Geneve: WHO; 2013.

19. Hoffmann RHS, Sousa MLR, Cypriano S. Prevalence of enamel defects and the relationship to dental caries in primary and permanent dentition, Indaiatuba, Sao Paulo, Brazil. Cad Saude Publica. 2007;23(2):435-44. http://dx.doi.org/10.1590/S0102-311X2007000200020. PMid:17221093.

20. Farsi N. Developmental enamel defects and their association with dental caries in preschoolers in Jeddah, Saudi Arabia. Oral Health Prev Dent. 2010;8(1):85-92. PMid:20480059.

21. Seow WK, Ford D, Kazoullis S, Newman B, Holcombe T. Comparison of enamel defects in the primary and permanent dentitions of children from a low fluoride District in Australia. Pediatr Dent. 2011;33(3):207-12. PMid:21703072.

22. Li Y, Navia JM, Bian J. Prevalence and distribution of developmental enamel defects in primary dentition of Chinese children 3-5 years old. Community Dent Oral Epidemiol. 1995;23(2):72-9. http://dx.doi. org/10.1111/j.1600-0528.1995.tb00204.x. PMid:7781303.

23. Vallejos-Sánchez AA, Medina-Solís CE, Casanova-Rosado JF, Maupomé G, Casanova-Rosado AJ, MinayaSánchez M. Enamel defects, caries in primary dentition and fluoride sources: relationship with caries in permanent teeth. Gac Sanit. 2007;21(3):227-34. PMid:17565898.

24. Targino AGR, Rosenblatt A, Oliveira AF, Chaves AMB, Santos VE. The relationship of enamel defects and caries: a cohort study. Oral Dis. 2011;17(4):420-6. http://dx.doi.org/10.1111/j.1601-0825.2010.01770.x. PMid:21114593.

25. Milgrom P, Riedy CA, Weinstein P, Tanner AC, Manibusan L, Bruss J. Dental caries and its relationship to bacterial infection, hypoplasia, diet, and oral hygiene in 6- to 36-month-old children. Community Dent Oral Epidemiol. 2000;28(4):295-306. http://dx.doi.org/10.1034/j.1600-0528.2000.280408.x. PMid:10901409.

26. Carvalho JC, Silva EF, Gomes RR, Fonseca JAC, Mestrinho HD. Impact of enamel defects on early caries development in preschool children. Caries Res. 2011;45(4):353-60. http://dx.doi.org/10.1159/000329388. PMid:21778723.

27. Cruvinel VR, Gravina DB, Azevedo TD, Rezende CS, Bezerra AC, Toledo OA. Prevalence of enamel defects and associated risk factors in both dentitions in preterm and full term born children. J Appl Oral Sci. 2012;20(3):310-7. http://dx.doi.org/10.1590/S1678-77572012000300003. PMid:22858696.

28. Seow WK. Environmental, maternal, and child factors which contribute to early childhood caries: a unifying conceptual model. Int J Paediatr Dent. 2012;22(3):157-68. http://dx.doi.org/10.1111/j.1365263X.2011.01186.x. PMid:21972925.

29. Listl S, Galloway J, Mossey PA, Marcenes W. Global economic impact of dental diseases. J Dent Res. 2015;94(10):1355-61. http://dx.doi.org/10.1177/0022034515602879. PMid:26318590.

30. Torriani DD, Ferro RL, Bonow MLM, Santos IS, Matijasevich A, Barros AJ, et al. Dental caries is associated with dental fear in childhood: findings from a birth cohort study. Caries Res. 2014;48(4):263-70. http:// dx.doi.org/10.1159/000356306. PMid:24503491. 